\title{
ESTUDO DA UTILIZAÇÃO DE MEDICAMENTOS ADMINISTRADOS POR SONDAS EM UM HOSPITAL UNIVERSITÁRIO
}

\author{
STUDY ON THE USE OF DRUGS ADMINISTERED VIA ENTERAL FEEDING TUBES IN A UNIVERSITY \\ HOSPITAL
}

Ione Cristina de Meneses Evangelista ${ }^{1}$, Jeamile Lima Bezerra²

1 Farmacêutica, Residente Multiprofissional em Alta Complexidade da Universidade Federal do Piauí. E-mail: ionecmeneses@gmail.com

${ }^{2}$ Dra., Prof.a Orientadora, Hospital Universitário da Universidade Federal do Piauí. E-mail: jeamilebezerra@yahoo.com.br

\section{ABSTRACT}

OBJECTIVE: To analyze standard oral medications in a university hospital, according to the possibility of being given via enteral feeding tubes. For this, an analysis was made of all oral drugs standardized in the institution according to the possibility of being administered through enteral catheter observing in literature the recommendations for manipulation and administration. METHODS: The study was carried out by means of a survey of standard oral medications in the hospital and subsequent review of the published literature, drug monograph, manufacturer's information, pharmacotechnical data of the pharmaceutical form, active principles and excipients. In addition, the study analyzed the prescriptions of patients who used medications via enteral tubes, tracing the consumption profile of the hospital and verifying if there is coherence between the prescriptions and the recommendations in literature. RESULTS: A total of 113 drugs and 3353 doses of oral medications were tested. It has been found that $40.7 \%$ of the oral drugs present in the standardization of the hospital are medications that can be administered by this route, but have some restriction in the manipulation and administration process and that they represent $50 \%$ of all medications administered by this route at the hospital. CONCLUSION: The study showed the importance of the professionals' knowledge involved in this practice regarding the information that is already available in literature on the use of probe and also exposed the problem of the scarcity of liquid presentations available in the market.

Keywords: Off-label use. Enteral nutrition. Routes of medication administration. Management of patient care.

\section{RESUMO}

Objetivo: Analisar os medicamentos orais padronizados em um hospital universitário, de acordo com a possibilidade de serem administrados por sonda enteral. Para isso, foi feita uma análise de todos os fármacos de uso oral padronizados na instituição de acordo com a possibilidade de serem administrados via sonda enteral observando na literatura as recomendações para manipulação e administração. Métodos: Estudo realizado por meio de levantamento dos medicamentos orais padronizados no hospital e posterior análise da revisão da literatura publicada, monografia das drogas, informação do fabricante, dados farmacotécnicos da forma farmacêutica, princípios ativos e excipientes. Além disso, o estudo analisou as prescrições de pacientes que 
faziam uso de medicações por essa via traçando o perfil de consumo do hospital e verificando se existe uma coerência entre as prescrições e as recomendações presentes na literatura. Resultados: Foram analisados 113 medicamentos e 3353 doses de medicamentos orais via sonda. Verificou-se que $40,7 \%$ dos fármacos orais presentes na padronização do hospital são medicações que podem ser administradas por esta via, mas possuem alguma restrição no processo de manipulação e administração e que elas representam $50 \%$ de todas as medicações feitas por essa via no hospital. Conclusão: O estudo mostrou a importância do conhecimento dos profissionais envolvidos nessa prática a respeito das informações que já estão disponíveis na literatura sobre o uso via sonda e expôs a problemática da escassez de apresentações líquidas disponíveis no mercado.

Palavras-chave: Uso Off-label. Nutrição Enteral. Vias de administração de medicamentos. Administração dos cuidados ao paciente.

\section{Como citar este artigo:}

Evangelista ICM, Bezerra JL. Estudo da utilização de medicamentos administrados por sondas em um Hospital Universitário. J. Ciênc. Saúde [internet]. 2018 [acesso em: dia mês abreviado ano];1(3):39-48. Disponível em: DOI: 


\section{INTRODUÇÃO}

A impossibilidade de fornecer nutrientes necessários para atender às exigências metabólicas sempre foi uma preocupação no também ambiente hospitalar em se tratando de pacientes clínicos e cirúrgicos. Pessoas com doenças crônicas, como acidente vascular cerebral e câncer ou ferimentos traumáticos estão particularmente em risco, assim como os idosos, que são especialmente vulneráveis às complicações decorrentes da desnutrição(1).

Quando os pacientes são incapazes de comer o suficiente para suprir suas necessidades nutricionais por mais de alguns dias, a terapia nutricional deve ser considerada. A nutrição enteral deve ser o primeiro tipo de nutrição a ser considerado. Se o trato gastrintestinal está em funcionamento, a terapia de nutrição enteral é uma forma de recuperar ou, em casos de danos repentinos, de manter um estado nutricional ótimo ${ }^{(2)}$.

Terapia nutricional enteral (TNE) é um conjunto de procedimentos cujo objetivo é manter e/ou recuperar o estado nutricional do paciente, por meio de via oral, sondas ou ostomias, através do fornecimento de energia e nutrientes ${ }^{(3)}$.

A TNE é uma alternativa usada frequentemente para a manutenção do estado nutricional ótimo de pacientes impossibilitados de se alimentar pela via oral de forma adequada ou quando o aporte nutricional total que pode receber pela via oral é insuficiente. Entretanto, na maioria das vezes, a sonda não é exclusiva para administração da nutrição enteral, sendo utilizada também para administração de medicamentos ${ }^{(4)}$.

Quando os pacientes que fazem uso de sondas de alimentação enteral não apresentam deglutição eficaz e correm riscos de aspiração pulmonar, as sondas de nutrição também são utilizadas para a administração de medicamentos, sendo este um procedimento de rotina na prática hospitalar. A via enteral para administração de fármacos tem vantagens evidentes, como a ampla disponibilidade de medicamentos para uso oral, baixo custo, diminuição do tempo de hospitalização e ausência de riscos associados à administração intravenosa, intramuscular, subcutânea e intradérmica ${ }^{(5)}$.

O uso de um medicamento para uma indicação não aprovada pela autoridade sanitária constitui o que chamamos de uso "off label'. Como a maioria das formas farmacêuticas sólidas não foi desenvolvida para ser administrada via ostomias, esta prática é considerada off label. Entretanto entende-se que esse uso não é incorreto se não expresso claramente a contraindicação pelo fabricante, sendo uma prática que apresenta poucos ou nenhum estudo de eficácia e segurança e que, portanto, responsabiliza os profissionais envolvidos nesse processo(6).

Outro problema relatado na literatura quando se opta por essa via de administração de medicamentos é a obstrução das sondas. Segundo Belknap ${ }^{(5)}$, as oclusões das sondas de nutrição enteral podem ocorrer em mais de $15 \%$ dos pacientes. Entre os eventos adversos dessa intercorrência são mencionados o tempo de envolvimento da enfermagem, a redução da tolerância dos pacientes a alimentos e medicamentos, a possiblidade de traumas e o aumento dos custos de internação.

Assim, a forma de preparo do medicamento para ser administrado via sonda, bem como a técnica usada na sua administração, impactam a efetividade do tratamento farmacoterapêutico. Há uma ampla variedade de procedimentos a serem aplicados ${ }^{(7,8)}$, mas a técnica deve sempre visar a redução das perdas quantitativas do fármaco durante o processo, bem como a manutenção da atividade do fármaco através de práticas como proteção do preparado da luz, administração imediata após o preparo, e solubilização em volume e solvente adequado. Também é importante que medicamentos diferentes sejam preparados e administrados separadamente para evitar a ocorrência de possíveis interações físico-químicas entre eles. A lavagem da sonda com água antes e após cada procedimento e a administração lenta do preparado também são essenciais para otimizar a absorção e ação do fármaco administrado via sonda ${ }^{(9)}$.

Tendo em vista os problemas citados, torna-se de grande importância focalizar atenção diferenciada ao 
tratamento farmacoterapêutico de pacientes sob o aporte de nutrição enteral e implementar medidas que aumentem a qualidade da administração de medicamentos via sonda. Desta forma, a administração de medicamentos por essa via sem uma análise do ponto de vista farmacológico e farmacotécnico pode gerar uma falha terapêutica e a perda da sonda enteral, um risco biológico para os profissionais de saúde e possíveis danos para o paciente. O objetivo do estudo foi traçar o perfil dos fármacos administrados via cateteres enterais em um hospital universitário e a partir do perfil, analisar os medicamentos orais padronizados na instituição de acordo com a possibilidade de serem administrados via sonda enteral e as recomendações para administração.

\section{METODOLOGIA}

A pesquisa trata-se de um estudo transversal, retrospectivo e descritivo, realizada entre maio e julho de 2017 de um Hospital Universitário de Teresina - PI. O objeto do estudo foi a investigação de medicamentos preparados e administrados por sonda enteral em pacientes dos 4 postos de internação do hospital, com base em recomendações encontradas na literatura.

A partir da consulta ao Aplicativo de Gestão para Hospitais Universitários (AGHU) do HU-UFPI, realizou-se um levantamento de todos os fármacos de uso oral (comprimidos, drágeas, cápsulas e soluções), da padronização da instituição e, após, análise destes acerca da possibilidade de utilização por sonda enteral, mediante revisão na literatura, por meio de busca eletrônica às bases de dados Portal de Periódicos CAPES/MEC e Scielo - Scientific Electronic Library Online, com publicações entre 2007 e 2017. Foram selecionados os trabalhos com propostas semelhantes a esta, sendo que alguns artigos encontrados serviram como fonte para buscas posteriores, por intermédio de suas referências. Nos casos em que as publicações supracitadas não continham informações ou estas eram insuficientes, a busca foi realizada através de monografias e livros.

Através do relatório diário fornecido pela equipe do EMTN (Equipe Multiprofissional de Terapia Nutricional) do hospital acerca dos pacientes submetidos a terapia nutricional, foram selecionados apenas aqueles pacientes em uso de suporte enteral. Todos os pacientes dentro desse grupo tiveram suas prescrições analisadas através do AGHU. Critérios de inclusão: todos os pacientes em uso de suporte enteral que estivessem em um dos quatro postos de internação do hospital e que em suas prescrições houvesse pelo menos um medicamento oral a ser administrado via sonda entre maio e julho de 2017. Critérios de exclusão: pacientes da Unidade de Terapia Intensiva e pacientes em uso de suporte enteral que não estavam recebendo medicamentos orais via sonda. Cada medicamento via sonda administrado no paciente correspondeu a uma dose de medicamento, assim ao total foram analisadas 3353 doses de medicamentos.

A partir disso, foi feita uma análise minuciosa de cada uma das 3353 doses de medicamentos preparados e administrados por sonda enteral para identificação do perfil destes. Para a análise dos dados foram utilizados procedimentos de estatística descritiva (média e percentagens) com a utilização do software Epi-Info para a tabulação e análise. Posteriormente esses dados foram apresentados em forma de gráfico.

Este projeto foi submetido à aprovação da Comissão de Aprovação de Projeto de Pesquisa (CAPP) do HU-UFPI e depois submetido ao Comitê de Ética em Pesquisa com Seres Humanos (CEP) por meio da Plataforma Brasil e aprovado com CAAE no 78789517.3.0000.5214.

\section{RESULTADOS}

Foram analisados 113 medicamentos orais da padronização do hospital: 50 comprimidos simples, 41 comprimidos revestidos, 4 comprimidos de liberação prolongada, 1 comprimido mastigável, 1 comprimido sublingual, 10 cápsulas, 4 soluções e 2 pós para dispersão.

Esses 113 fármacos foram divididos em quatro categorias: fármacos que podem ser triturados, fármacos que podem ser triturados com restrição, fármacos que podem ser diluídos e fármacos que não podem ser triturados nem diluídos.

A partir da análise feita, verificou-se que desses 113 medicamentos presentes na padronização do hospital, 
35 (31\%) são medicamentos que podem ser triturados sem restrições; 46 (40,7\%) são medicamentos que podem ser administrados por esta via, mas possuem alguma restrição no processo de manipulação e administração; 13 (11,5\%) são medicamentos que não podem ser triturados, mas podem ser diluídos e 19 $(16,8 \%)$ são medicamentos que não devem ser triturados nem diluídos e requerem substituição ou análise risco-benefício no tratamento do paciente (Gráfico 1.0).

\section{Gráfico 1.0: Categoria de medicamentos.}

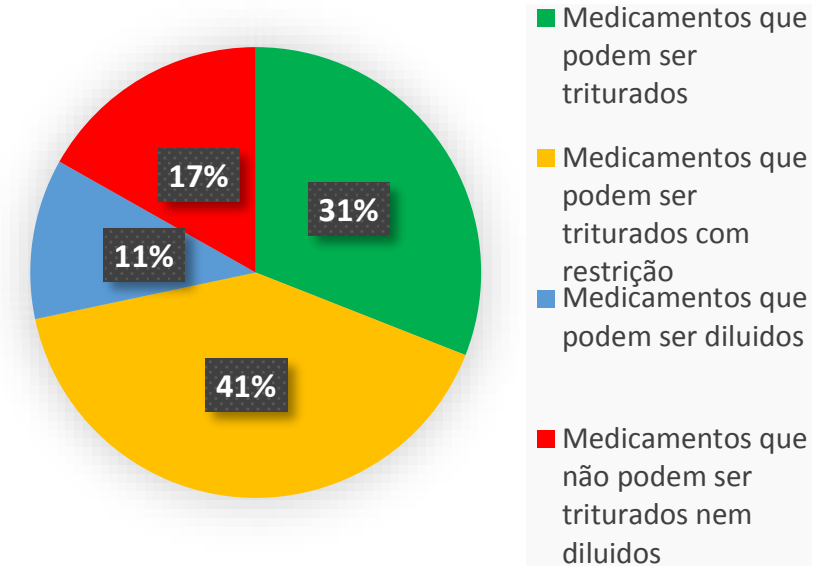

Analisando o perfil do consumo de medicamentos orais via sonda no hospital, observou-se que das 3353 doses de medicamentos analisadas, 1169 (35\%) são de medicamentos que podem ser triturados, $288(9 \%)$ são de medicamentos que podem ser diluídos, 205 (6\%) são de medicações que não podem ser trituradas nem diluídas, e, a maioria, 1691 (50\%) são medicações que podem ser trituradas mas que apresentam alguma particularidade na sua manipulação ou administração (Gráfico 2.0).

\section{Gráfico 2.0. Perfil do consumo de medicamentos via sonda.}

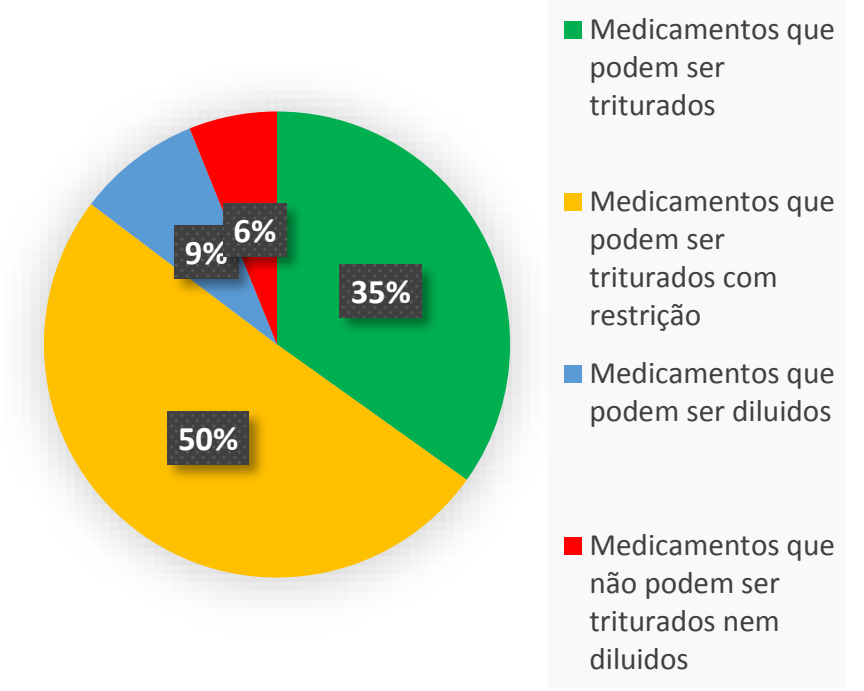

Analisando as prescrições foi possível identificar as 20 medicações mais utilizadas no hospital via sonda enteral. Elas representaram cerca de $63 \%$ de todas as medicações orais consumidas via sonda nas prescrições analisadas. A grande maioria compreende medicações que agem no sistema nervoso central, sistema cardiovascular e sistema digestivo. De acordo com a literatura, nenhum destes medicamentos foi classificado como "não triturável" e, portanto, poderiam ser administrados por cateteres enterais.

\section{Gráfico 3. Principais medicamentos orais utilizados via sonda no HU-UFPI.}

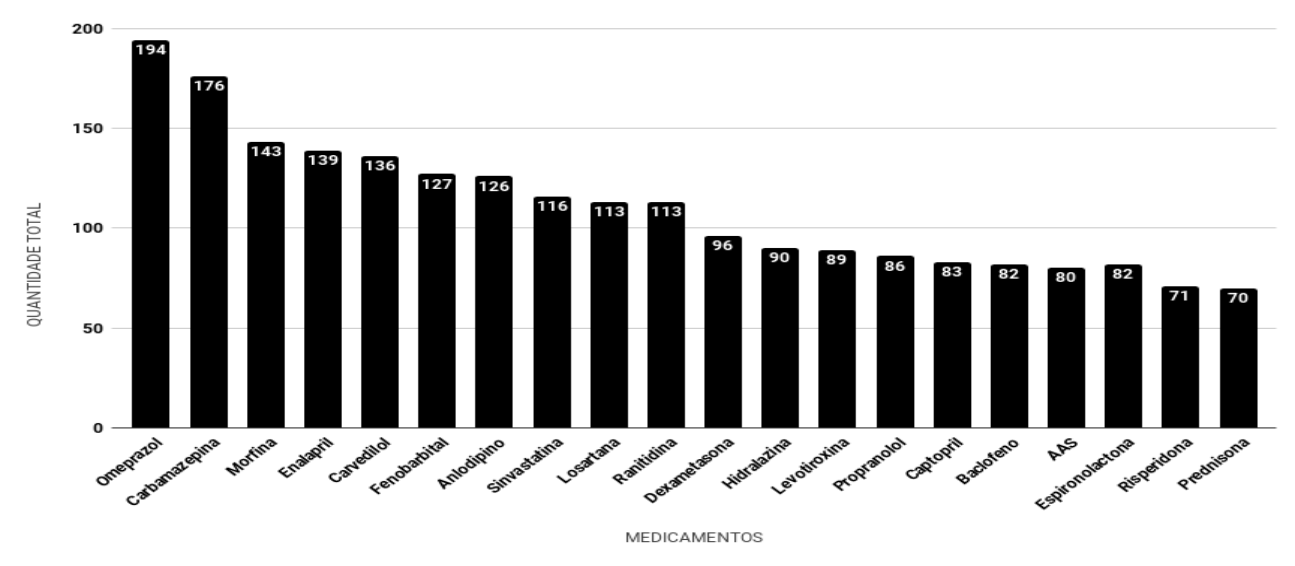




\section{DSICUSÃO}

De modo geral, as formas farmacêuticas mais indicadas para administração através de cateter enteral são as preparações líquidas como soluções, suspensões, xaropes e elixires. Para outras formulações como comprimidos, grânulos, cápsulas e drágeas deve-se analisar o risco de alteração do princípio ativo e deve-se considerar ainda que o risco de obstrução do cateter é maior. Desta forma, sempre que possível, recomendase substituir a forma sólida por preparações líquidas manipuladas, para evitar o risco de obstrução e perda da eficácia do medicamento. Por isso é importante que os hospitais - e principalmente hospitais de alta complexidade - disponham de um setor de Farmacotécnica, visto que na ausência de formulações líquidas para substituição, alguns autores recomendam a administração de formulações extemporâneas, que são mais estáveis, produzidas a partir do próprio medicamento. O custo com o preparo dessas formulações seria relativamente o mesmo, ou até um pouco mais elevado do que a adaptação realizada atualmente, devido ao consumo de excipientes como: agentes de suspensão, conservantes, agentes antioxidantes, entre outros. Entretanto, haveria vantagem não só na redução do tempo gasto pela equipe de enfermagem no preparo dos medicamentos adaptados - podendo se dedicar mais às atividades assistenciais aos pacientes - como também tais formulações seriam manipuladas pelo próprio farmacêutico, que é conhecedor das formas farmacêuticas. Essas formulações extemporâneas poderiam ser preparadas em quantidades maiores e armazenadas por mais tempo, a depender da estabilidade alcançada ${ }^{(10,11)}$.

O HU-UFPI atualmente não dispõe do setor de farmacotécnica, neste caso, a padronização de alternativas líquidas seria o ideal por se tratar de um hospital de alta complexidade, com grande volume de pacientes que sofrem de problemas crônicos que impedem a administração de medicamentos por via oral.
Neste estudo verificou-se que a forma farmacêutica mais utilizada para essas adaptações foram os comprimidos que, frequentemente, podem ser triturados e administrados via sonda, pois há pouca diferença no tempo de absorção e concentração plasmática em relação à utilização do comprimido na sua forma íntegra. Para comprimidos de liberação controlada, a trituração altera seu perfil de liberação e afeta significativamente seu efeito farmacológico. O revestimento entérico evita que o fármaco seja inativado pelo $\mathrm{pH}$ gástrico e previne danos ao trato gastrointestinal através do retardamento do início da ação. Esta cobertura assegura a estabilidade e efetividade do fármaco e, portanto, medicações como o bisacodil, metildopa, mesalasina, prometazina, sulfasalazina, sulfato ferroso, topiramato e vitamina B não devem ser trituradas. Além disso, o revestimento do fármaco dificulta a trituração, formando precipitados que se agregam à parede da sonda, podendo obstruí-la $a^{(12,13)}$.

Fármacos de ação modificada objetivam garantir que a sua liberação ocorra por um período prolongado de tempo e de forma controlada. Entre estes, estão os pellets, sistemas multiparticulados revestidos, obtidos através de procedimentos tecnológicos que apresentam melhorias na biodisponibilidade e na segurança da liberação do fármaco, todavia sua trituração ocasiona perda de suas propriedades e, como consequência, de seu efeito terapêutico. É o caso das medicações padronizadas no hospital (diltiazem, metoprolol, nifedipino e a oxicodona) que são comprimidos e possuem um revestimento de liberação prolongada que ao ser triturado possui risco de toxicidade e manutenção inadequada do nível sérico do fármaco e, portanto, não deve ser triturado.

A principal característica dos fármacos de uso sublingual é a rápida dissolução e absorção por meio dos fluidos orais. A isossorbida sublingual, por exemplo, quando administrada via oral, sofre alteração na biodisponibilidade e intenso metabolismo de primeira passagem, fatores que comprometem sua eficácia, restringindo assim seu uso à via sublingual(11).

De modo geral, cápsulas de gelatina dura podem ser abertas e seu conteúdo em forma de pó diluído em 15 
a $30 \mathrm{~mL}$ de água destilada para administração por sonda enteral. Para evitar oxidação e perdas devido à umidade, cápsulas e comprimidos de liberação imediata devem ser administrados logo após abertura e trituração/dissolução, respectivamente ${ }^{(1)}$.

Algumas cápsulas de gelatina dura podem apresentar o princípio ativo na forma de microgrânulos, os chamados pellets, que consistem no agrupamento de pós finos de substância ativa e excipientes em pequenas unidades esféricas $^{(14)}$. É o caso de fármacos como itraconazol, tramadol, gabapentina, amoxicilina, fluconazol e fluoxetina. Nesses casos, os pellets não devem ser triturados e as cápsulas podem ser abertas e o conteúdo dissolvido em água imediatamente antes da administração. O omeprazol que, de acordo com a análise, foi a medicação mais utilizada via sonda, também não pode ser triturado, mas pode ser diluído. Diferentemente dos últimos citados, ele necessita de técnica de preparo diferenciada dependendo do local de inserção da sonda. Se a posição da sonda for póspilórica, recomenda-se diluir em bicarbonato de sódio $8,4 \%$ antes da administração, o que proporciona atividade de tampão e o protege da inativação do $\mathrm{pH}$ ácido. Se a posição da sonda for gástrica, recomenda-se diluir os pellets em suco ácido (maçã, laranja e outros) antes da administração(11,15).

Medicamentos que são teratogênicos, carcinogênicos, citotóxicos, hormônios, análogos de prostaglandina e os potencialmente alergênicos, em virtude do risco ocupacional, nunca devem ser triturados, pois o pó liberado durante a trituração pode expor os manipuladores dos medicamentos a riscos. Esse procedimento deve ser realizado em capela de fluxo laminar e com paramentação adequada ${ }^{(6,1)}$.

As soluções injetáveis são de alto custo, porém podem ser utilizadas quando o princípio ativo não tem ação devido ao $\mathrm{pH}$ estomacal. Contudo, deve-se considerar as divergências de biodisponibilidade com outras formulações, além do fato de que precisam ser diluídas em virtude da alta osmolaridade ${ }^{(6)}$.

Analisando o perfil de medicamentos utilizados no hospital via sonda (Gráfico 2), verificou-se que metade dos medicamentos consumidos nas prescrições analisadas (50\%), são de medicações que podem ser trituradas, mas que possuem restrições na sua manipulação ou na sua administração. A revisão bibliográfica mostrou que ainda há carência de recomendações nacionais sobre o preparo e administração dos medicamentos por cateteres enterais, inclusive nas suas bulas e que a maioria das informações disponíveis é proveniente de trabalhos realizados em outros países. Isso mostra a importância do conhecimento dos profissionais envolvidos nessa prática a respeito das formas farmacêuticas e das informações que já estão disponíveis na literatura sobre o uso via sonda, não somente com relação à possibilidade ou não de trituração, mas também outros fatores que influenciam no sucesso da terapia por essa via, como intervalo de tempo entre dieta e medicamentos e interação dieta-alimento.

Quando um comprimido é administrado mesmo havendo a possibilidade de interação com alimento, esta pode ser minimizada pelo ajuste do aprazamento de horário da medicação em relação ao horário das refeições. $O$ intervalo de tempo adequado entre administração de medicamentos e alimentos ajuda na diminuição de possíveis interações entre estes e otimiza a absorção dos fármacos, portanto, exige-se um planejamento adequado quanto ao aprazamento dos fármacos e da dieta. O atelonol, captopril, carbamazepina, domperidona, hidroclorotiazida, levotixoxina, entre outros, na presença de alimentos pode diminuir a resposta farmacoterapêutica pela redução da sua absorção. Fármacos como ciprofloxacino, clorpromazina, fenitoina, levofloxacino e norfloxacino na presença de alimentos podem formar quelatos devido à complexação com íons presentes na nutrição, reduzindo ou aumentando significativamente seu efeito farmacológico e até mesmo inativando o princípio ativo, necessitando que estes sejam administrados com intervalo de uma hora antes ou duas horas após a dieta. Já para os fármacos em que a dieta não influencia na absorção, é recomendado que a nutrição seja interrompida e que seja feita a lavagem da sonda antes e depois da administração da medicação ${ }^{(16,17,18)}$. 
Outro importante ponto quando se fala sobre a administração de medicamentos via cateteres enterais é a obstrução da sonda. A oclusão da sonda pode ocorrer por vários fatores: administração de fármacos, incompatibilidades fármaco-fármaco e fármaconutriente, formulação da dieta, regime de administração e calibre da sonda. O procedimento de desobstrução pode resultar em perdas farmacológicas e nutricionais, além de gastos com troca da sonda, tempo dos profissionais envolvidos e também levar a traumas para o paciente. Sendo assim, cuidados como lavar adequadamente a sonda com 20 a $30 \mathrm{~mL}$ de água destilada, utilizando seringa para aplicar uma leve pressão na lavagem antes e após a administração de medicamentos influencia diretamente no seu tempo de uso e, consequentemente, na durabilidade $\mathrm{e}^{(19,12,1)}$.

No estudo verificou-se que das 20 medicações mais utilizadas via sonda no hospital (Gráfico 3), a grande maioria são medicações que agem no sistema nervoso central e sistema cardiovascular, medicações que normalmente não estão disponíveis na forma injetável ou que não existem formas alternativas líquidas. Isso nos faz perceber que há certo desinteresse por parte da indústria de medicamentos na produção de formulações orais líquidas de determinadas classes farmacológicas, o que pode estar relacionado diretamente com o fato de que tais formulações são, em sua maioria, destinadas a pacientes pediátricos. Outro fator determinante seria a preferência pela produção de formas farmacêuticas sólidas, uma vez que estas são mais estáveis e apresentam um maior tempo de validade quando comparadas com apresentações líquidas ${ }^{(10)}$.

A justificativa fundamental para a necessidade da trituração de comprimidos é a indisponibilidade no mercado de formulações líquidas de determinados fármacos essenciais na terapia farmacológica dos pacientes. A carência de formulações líquidas gera, inevitavelmente, a demanda por adaptações de formas farmacêuticas sólidas ${ }^{(10)}$.

A maior disponibilidade de formas líquidas na instituição poderia beneficiar não somente os pacientes em TNE, como também aqueles com queixas de disfagia, odinofagia e pacientes com megaesôfago, situações comumente observadas em hospitais de alta complexidade $^{(20)}$. Algumas adaptações poderiam ser evitadas pela padronização de apresentações líquidas disponíveis no mercado de alguns fármacos. Carbamazepina e fenobarbital, que como o estudo demonstrou (Gráfico 3) são medicações bastante utilizadas em adaptações e que poderiam ser substituídas pelas suas respectivas soluções orais disponíveis no mercado. A comissão de padronização do hospital deve ser responsável por selecionar medicamentos a serem utilizados na instituição que atendam ao máximo as necessidades do corpo clínico desta.

Os profissionais envolvidos na administração de medicamentos por sonda têm um papel muito importante e devem, primeiramente, avaliar a farmacoterapia do paciente, a necessidade de utilização de determinado medicamento e se é possível ou não ser administrado por esta via. Por isso, é essencial conhecer as formas farmacêuticas e dosagens disponíveis dos medicamentos no hospital - a fim de realizar a intercambialidade da apresentação farmacêutica, quando necessário -, bem como que se faça um treinamento adequado quanto à manipulação e administração desses medicamentos, visto que essa prática é pouco estudada durante sua formação.

A demanda por adaptações para cateteres enterais sempre irá existir nos hospitais e a participação do farmacêutico como membro da equipe de terapia nutricional é essencial para evitar problemas relacionados com medicamentos nos pacientes em TNE. Ele deve ser responsável por avaliar a adequabilidade dessas adaptações e propor soluções que viabilizem procedimentos mais seguros e eficazes, garantindo assim o sucesso da terapia nutricional e farmacológica ${ }^{(10,1)}$.

\section{CONCLUSÃO}

A utilização da via enteral para aporte de fármacos é uma prática comum nos hospitais quando o paciente se encontra em TNE. Embora a grande maioria dos fármacos sólidos orais possa ser administrada por cateteres enterais, a padronização e escolha na hora da 
prescrição de alternativas viáveis para substituição, possibilita aos pacientes e aos profissionais, maior segurança e comodidade na administração. Quando nesse processo ocorrem falhas, isso pode trazer consequências danosas ao paciente e à instituição, como: desconforto (desobstrução e repassagem de sonda), comprometimento do tratamento, além de elevar os custos envolvidos nesse processo. 0 conhecimento adequado sobre a farmacotécnica dos fármacos e a compatibilidade fármaco-nutriente também auxilia no êxito da farmacoterapia.

Assim, considerando a carência de estudos e referências bibliográficas que tratem da administração de medicamentos orais por cateteres enterais, este trabalho constitui uma ferramenta fundamental para auxiliar e assegurar a qualidade do processo de cuidado farmacoterapêutico e êxito da terapia medicamentosa prescrita para os usuários destas sondas, por meio da elaboração de um manual de apoio personalizado às necessidades do hospital e deve ser utilizado como uma ferramenta auxiliar para o conhecimento dos principais problemas e complicações da administração de medicamentos via sonda.

A administração de medicações via sonda pode ser uma aliada na farmacoterapia de pacientes em TNE desde que feita da maneira correta e seguindo as recomendações da literatura. Por isso, intervenções farmacêuticas se tornam indispensáveis na resolução de problemas relacionados à farmacoterapia, reduzindo possíveis reações adversas, interações entre fármacos e alimentos, promovendo a qualidade do tratamento dos pacientes, facilitando o trabalho dos profissionais envolvidos no cuidado e reduzindo custos desnecessários.

\section{REFERÊNCIAS}

1. Heydrich J. Padrão de prescrição, preparo e administração de medicamentos em usuários de sonda de nutrição enteral. 2006. 108p. Dissertação de mestrado. Faculdade de Farmácia, Universidade Federal do Rio Grande do Sul, Porto Alegre.
2. Krause. Alimentos, nutrição e dietoterapia/ editado por L. Kathlen Mahan, Sylvia Escott-Stump. 10. ed. São Paulo: Roca, 2002. 1157 p.: il.

3. Waitzberg DL. Nutrição oral, enteral e parenteral na prática clínica. 3ed. São Paulo: Editora Atheneu, 2000. 928p.

4. ISMP. Preparo e administração de medicamentos via sonda enteral ou ostomias, 2005. [Acesso em: 29 abr. 2017]. Disponível em: http://proqualis.net/boletim/preparo-eadministraçao-de-medicamentos-sonda-enteral-ouostomias

5. Belknap DC, Seifert CF, Petermann M. Administration of medications through enteral feedings catheters. American Journal of Critical Care, 1997;6(5):382-92.

6. Moriel P, Shoji P, Bortoletto TC, et al. Uso Off Label De Medicamentos Através de Sondas:

Divergência Entre Informações. Rev. Bras. Farmácia Hospitalar e Serviços Saúde. 2012;3(2):20-4.

7. Matsuba CST, Gutiérrez MGR, Whitaker IY. Development and Evaluation of Standardized Protocol to Prevent Nasoenteral Tube Obstruction in Cardiac Patients Requiring Enteral Nutrition with Restricted Fluid Volumes. Journal of Clinical Nursing. 2007;10(16):1872-77.

8. Phillips NM, Nay R. A Systematic Review of Nursing Administration of Medication via Enteral Tubes in Adults. Journal of Clinical Nursing.

2008;17(17):2257-65.

9. Gilbar PJ. A Guide to Enteral Drug

Administration in Palliative Care. Journal of Pain and Symptom Management. New York, 1999;7(3):197-207.

10. Nunes MS, et al. Análise das solicitações de comprimidos adaptados para pacientes críticos de um hospital universitário. Revista Brasileira de Farmácia Hospitalar e Serviços de Saúde, São Paulo, 2013;4(4)18-25. 
11. Jamal Y, Dumke EH. Padronização de medicamentos sólidos orais via sonda nasoenteral em um hospital em Cascavel, Paraná. Revista Thêma at Scientia, 2012;2(2):91-106,.

12. Gorzoni ML, Della Torre A, Pires, SL. Medicamentos e sondas de nutrição. Revista da Associação Médica Brasileira, São Paulo, 2010;56(1):17-21.

13. Lima G, Negrini NMM. Assistência farmacêutica na administração de medicamentos via sonda: escolha da forma farmacêutica adequada.

Einstein, São Paulo, 2009;7(1):9-17.

14. Santos HMM, Veiga FJB, Pina MET, Souza, JJMS. Obtenção de pellets por extrusão e esferonização farmacêutica - Parte I - Avaliação das variáveis tecnológicas e de formulação. Rev. Bras. Cienc. Farm., Dez 2004;40(4):455-70.

15. Ferracini FT, Almeida SM, Filho WMB. Material de apoio para Farmácia Clínica. In: Ferracini, FT; Filho, WMB. (Org.). Farmácia Clínica: Segurança na prática hospitalar. São Paulo: Atheneu, 2011. p. 368-73.

16. Neto JFM. Farmácia Hospitalar e suas interfaces com a saúde. São Paulo: Rx, 2005. 316 p.
17. Farias $\mathrm{MA}$, et al. Estruturação de orientação farmacêutica para com medicamentos por sonda nasoenteral: um estudo de caso. Revista Brasileira de Farmácia, 2011;92(4):378-83.

18. Soares FK, et al. Protocolo de transformação e diluição de medicamentos para utilização por sonda de nutrição enteral. Fármaco, Ponta Grossa, 2012;1(3):5.

19. Carvalho AMR, et al. Análise da prescrição de pacientes utilizando sonda enteral em um hospital universitário do Ceará. Revista Brasileira de Farmácia Hospitalar e Serviços de Saúde, São Paulo, 2010;1(1):17-21.

20. Silva MFB, Brito PD, Guaraldo L. Medicamentos orais de uma unidade hospitalar: adequação ao uso por cateteres enterais. Rev. Bras. Enferm. [online]. 2016;69(5):847-54.

Sources of funding: No Conflict of interest: No Accepted: 2019/02/06

Publishing: 2019/12/24

Corresponding Address: Ione Cristina de Meneses Evangelista. Teresina, Piauí, Brasil. E-mail: ionecmeneses@gmail.com 